\title{
Perfusion Tissue Culture Initiates Differential Remodeling of Internal Thoracic Arteries, Radial Arteries, and Saphenous Veins
}

\author{
David A. Prim $^{\mathrm{a}}$ Vinal Menon $^{\mathrm{b}}$ Shahd Hasanian ${ }^{\mathrm{a}}$ Laurel Carter ${ }^{\mathrm{b}}$ \\ Tarek Shazly ${ }^{a, c}$ Jay D. Potts ${ }^{a, b}$ John F. Eberth ${ }^{a, b}$ \\ aBiomedical Engineering Program, College of Engineering and Computing, University of South Carolina, \\ Columbia, SC, USA; ${ }^{b}$ Department of Cell Biology and Anatomy, School of Medicine, University of \\ South Carolina, Columbia, SC, USA; ' ${ }^{C}$ Mechanical Engineering, College of Engineering and Computing, \\ University of South Carolina, Columbia, SC, USA
}

\section{Keywords}

Vessel culture · Coronary artery bypass grafting · Perfusion bioreactor · Vascular remodeling $\cdot$ Vein graft

\begin{abstract}
Adaptive remodeling processes are essential to the maintenance and viability of coronary artery bypass grafts where clinical outcomes depend strongly on the tissue source. In this investigation, we utilized an ex vivo perfusion bioreactor to culture porcine analogs of common human bypass grafts: the internal thoracic artery (ITA), the radial artery (RA), and the great saphenous vein (GSV), and then evaluated samples acutely (6 h) and chronically (7 days) under in situ or coronary-like perfusion conditions. Although morphologically similar, primary cells harvested from the ITA illustrated lower intimal and medial, but not adventitial, cell proliferation rates than those from the RA or GSV. Basal gene expression levels were similar in all vessels, with only COL3A1, SERPINE1, $F N 1$, and TGFB1 being differentially expressed prior to culture; however, over half of all genes were affected nominally
\end{abstract}

by the culturing process. When exposed to coronary-like conditions, RAs and GSVs experienced pathological remodeling not present in ITAs or when vessels were studied in situ. Many of the remodeling genes perturbed at $6 \mathrm{~h}$ were restored after 7 days (COL3A1, FN1, MMP2, and TIMP1) while others (SERPINE1, TGFB1, and VCAM1) were not. The findings elucidate the potential mechanisms of graft failure and highlight strategies to encourage healthy ex vivo pregraft conditioning.

๑) 2018 S. Karger AG, Basel

\section{Introduction}

Coronary artery bypass grafts (CABGs) experience wide variations in failure rates depending on the source tissue used for the graft, with approximately $6 \%$ of 400,000 procedures per year experiencing some sort of failure in the first 12 months $[1,2]$. Although vascular cell activity is vital to healthy remodeling, graft failure is often the result of intimal hyperplasia, whereby a complex remodel-

\section{KARGER}

(c) 2018 S. Karger AG, Basel

E-Mail karger@karger.com

www.karger.com/jvr
Dr. John F. Eberth

USC SOM CBA

Bldg. 1, Rm. C-36

Columbia, SC 29208 (USA)

E-Mail john.eberth@uscmed.sc.edu 
ing cascade of differentiation, migration, and proliferation of smooth-muscle cells (SMC), extracellular matrix (ECM) protein deposition, and infiltration of circulating inflammatory and progenitor cells lead to the formation of an abnormal tissue layer between the intima and internal elastic lamina. This process eventually causes graft narrowing, restenosis, and occlusion, resulting in surgical reintervention [3-5]. An intuitive approach to improving clinical outcomes would therefore be to stimulate the inherent healthy processes in a graft tissue while attenuating maladaptive remodeling.

Throughout development, mechanosensitive vascular cells dictate the growth and organization of blood vessels in response to hemodynamic loads. The resulting mature vascular tissues are optimized with respect to their specific loading conditions [6-8]. While the number of CABG failures remains unacceptably high, for most current CABGs to be successful, one of the following must be true: (i) vessels can function effectively in loading conditions for which they may not be developmentally optimized, or (ii) vessels adapt to chronic changes to their environment. Other authors have reported long-term compensatory changes in grafts that are successful $[9,10]$, supporting the validity of (ii). This adaptation hypothesis assumes that vascular cells within graft vessels sense and respond to chronically altered mechanical loads; this has been theoretically accepted for decades and corroborated in a wide-range of experiments [11-18]. There is evidence to suggest that vascular cells in discrete vessels, arising from different embryological origins, do not function uniformly and respond differently to stimuli $[19,20]$. Thus, it is plausible that inherent differences between CABG vessels could affect their ability to successfully adapt to grafting.

To assess remodeling capability at a reasonable time scale, we can evaluate the expression of genes associated with vascular remodeling as a proxy for eventual growth and remodeling outcomes. Type III collagen (the COL3A1 gene), for example, is associated with the structural fibers contributing to the mechanical strength of the vessel wall as well as being a component of the basement membranes. Type III collagen deposition and remodeling are also associated with graft restenosis and intimal hyperplasia [21, 22]. Matrix metallopeptidase II (the MMP2 gene) degrades several types of collagen, and is inhibited by tissue inhibitor of metallopeptidase (the TIMP1 gene), both of which are known to exhibit altered expression in response to blood flow and pressure; therefore, altered expression of either of these genes could be an indicator of active vascular remodeling $[9,23]$. Fibro- nectin 1 (the FN1 gene) is an ECM component that has been shown to be activated by cyclic stretch and it also plays a role in SMC phenotype modulation, proliferation, and cell adhesion [24]. Plasminogen activator inhibitor type 1 (the SERPINE1 gene) is expressed by endothelial cells (EC) and SMC in response to both altered cyclic strain and flow, influences fibrinolytic balance, regulates ECM proteolysis, and can inhibit matrix degradation by inhibiting metallopeptidases (MMPs) [25]. Vascular cell adhesion molecule type 1 (the VCAM1 gene) is an endothelial marker that responds to flow and is implicated in inflammatory processes as a precursor to neointima formation [26]. Transforming growth factor $\beta 1$ (the TGFB1 gene) is implicated in numerous cardiovascular processes, specifically cell growth, proliferation, differentiation, and apoptosis, and its expression has been shown to change in response to altered cyclic strain $[27,28]$.

In this investigation, we measured and compared the early remodeling response of 3 common CABG tissues, the internal thoracic artery (ITA), radial artery (RA), and great saphenous vein (GSV), to coronary loading conditions by culturing samples in an ex vivo perfusion bioreactor. We hypothesized that inherent differences exist in each vessel's response to altered stimuli, and thus their ability to remodel in response to CABG. Further understanding of differential remodeling can then be used to guide future investigations into graft preconditioning optimization strategies.

\section{Materials and Methods}

Tissue Harvesting and Preparation

The left anterior descending coronary artery (LAD), ITA, RA, and GSV samples were dissected from freshly slaughtered American Yorkshire sows (approx. $200 \mathrm{~kg} ; 3$ years old) at a local abattoir. All tissues used in this study were acquired from animals designated for human consumption and therefore did not fall under the IACUC protocol. Prior to any experimental manipulation (time point $0, \mathrm{t} 0$ ), fresh samples were briefly rinsed in sterile Moscona's saline solution and then snap-frozen on dry ice. All other samples were excised and then transported in tubes containing sterile Moscona's saline solution supplemented with $20 \mathrm{U} / \mathrm{mL}$ heparin, $1 \%$ penicillin/streptomycin, $1 \%$ amphotericin-B, and $1 \%$ gentamycin. Upon arrival at the laboratory, vessels were cleaned of excess perivascular and adventitial tissue, branches ligated with sterile 6-0 silk suture, and samples were mounted on custom-made barbed glass cannulas within custom-made lidded glass bioreactor baths (Adams and Chittenden Scientific Glass, Berkeley, CA, USA). For the 7-day experiments, tissue sections adjacent to the mounted samples were fixed in $4 \%$ paraformaldehyde for later comparison to the cultured samples. 
Cell Isolation and Proliferation for Plated Studies

To isolate intimal cells, one end of each vessel was occluded with a sterile 3-0 silk suture. The lumen was then filled with $0.15 \%$ w/v collagenase II solution in serum-free DMEM (Worthington Biochemical, Lakewood, NJ, USA), followed by clamping of the open end of the vessel, and incubation for $8 \mathrm{~min}$ at $37^{\circ} \mathrm{C}$. After incubation, the collagenase solution within each vessel was decanted into $15-\mathrm{mL}$ centrifuge tubes, and DMEM supplemented with $10 \%$ FBS was rinsed through the lumen into a centrifuge tube to further dislodge intimal cells. A cut was then made along the length of the vessel so that the lumen could be gently scraped with a cell scraper to isolate any remaining intimal cells. These contents were centrifuged for $8 \mathrm{~min}$ at $800 \mathrm{rpm}$, resuspended in DMEM supplemented with $5 \%$ FBS, and plated for culture in dishes with collagen-coated coverslips. EC populations were confirmed at day 7 using immunofluorescence images of platelet endothelial adhesion molecule 1 (CD31/PECAM1) phalloidin, and 4',6-diamidino2-phenylindole (DAPI) (All antibodies: Abcam Inc., Cambridge, MA, USA) on a Zeiss LSM 510 confocal microscope (Carl Zeiss Microscopy GmbH, Jena, Germany).

Following intima removal, denuded vessels were placed in fresh tubes containing collagenase II solution (as described above) and incubated for $10 \mathrm{~min}$ at $37^{\circ} \mathrm{C}$ with mechanical agitation. After incubation, samples were removed from tubes, and the loosened adventitia removed with forceps and returned to the tubes of collagenase solution. DMEM supplemented with 10\% FBS was added to the tubes containing the adventitia and collagenase solution, and the tubes were centrifuged for $8 \mathrm{~min}$ at $800 \mathrm{rpm}$, followed by resuspension in fresh DMEM with 10\% FBS, and then plated for culture with collagen-coated coverslips. The presence of SMC was confirmed by confocal microscopy at day 7 using immunofluorescence images of fluorescently labeled $\alpha$-smooth-muscle actin ( $a$-SMA) antibodies and DAPI on the Zeiss confocal microscope.

After adventitia removal, the remaining tissues were placed in fresh tubes of collagenase solution and incubated with agitation for $30 \mathrm{~min}$ at $37^{\circ} \mathrm{C}$. Samples were then cut into small pieces (1-2 $\mathrm{mm}^{2}$ ), returned to tubes containing collagenase solution, and incubated further at $37^{\circ} \mathrm{C}$ for $4 \mathrm{~h}$ with agitation. Following incubation, DMEM with $10 \%$ FBS was added, and samples were centrifuged for $8 \mathrm{~min}$ at $800 \mathrm{rpm}$, resuspended in fresh DMEM with $10 \%$ FBS, and plated for culture with collagen-coated coverslips. The presence of fibroblasts was confirmed by confocal microscopy at day 7 using immunofluorescence images of fibroblast-specific protein 1 (FSP1/S100A4) and DAPI on the Zeiss confocal microscope.

Cell cultures were assessed qualitatively using an inverted phase-contrast microscope with a CCD camera. Proliferation was quantitated using the 5-bromo-2'-deoxy-uridine (BrdU) labeling and detection kit I (Roche Diagnostics GmbH, Mannheim, Germany) according to the manufacturer's instructions. Briefly, after 7 days of culture, $3 \mu \mathrm{L} \mathrm{BrdU/mL} \mathrm{medium} \mathrm{was} \mathrm{added} \mathrm{to} \mathrm{plates} \mathrm{con-}$ taining coated coverslips for $3 \mathrm{~h}$. After labeling, coverslips were rinsed 3 times in PBS, fixed in an ethanol/glycine fixative solution at $-20^{\circ} \mathrm{C}$ for $30 \mathrm{~min}$, and then rinsed 3 more times in PBS. For staining, coverslips were covered with $100 \mu \mathrm{L}$ anti-BrdU diluted $1: 10$ with incubation buffer (both from the Roche kit above) and incubated for $1 \mathrm{~h}$ at $37^{\circ} \mathrm{C}$, followed by 3 rinses for $10 \mathrm{~min}$ each in PBS. Next, coverslips were covered with fluorescein-labeled antimouse IgG diluted 1:10 in PBS and incubated for $1 \mathrm{~h}$ in the dark at $25^{\circ} \mathrm{C}$, followed by 3 rinses of $10 \mathrm{~min}$ each in PBS, staining with DAPI diluted 1:5,000 in PBS, and incubation for $1 \mathrm{~h}$ in the dark at

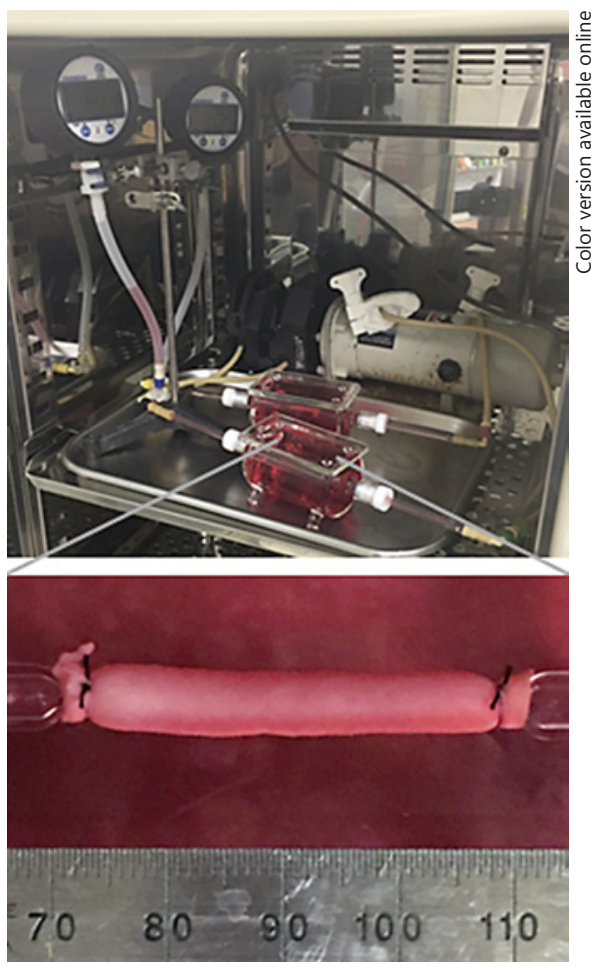

Fig. 1. Parallel ex vivo pulsatile perfusion bioreactors assembled in the incubator (top) with the ITA sample in culture (bottom).

room temperature. After rinsing, coverslips were mounted using a 1:1 PBS/glycerol mounting medium and imaged using the Zeiss confocal microscope.

\section{Ex vivo Vessel Culture}

The ex vivo bioreactor used for this investigation was designed so that most wetted parts could be easily autoclaved and contained in an incubator when assembled (Fig. 1). Pulsatile and steady flow were generated via a Masterflex L/S Easy-Load II roller pump head (model 77201-60) attached to a Masterflex L/S roller pump (model 7552-02), and output was controlled by a Masterflex wash-down modular controller (model 7552-71), all from Cole-Parmer (Vernon Hills, IL, USA). Pressure was adjusted via a tubing pinch valve downstream of the vessel and measured on a digital pressure gauge (model DPG8001) (Omega, Norwalk, CT, USA) located upstream of the sample offset. All tubing had an inner diameter of $0.64 \mathrm{~cm}$ (Masterflex PharMed BPT, Cole-Parmer). The temperature in the incubator was maintained at $37^{\circ} \mathrm{C}$ and the $\mathrm{CO}_{2}$ concentration was maintained at $5 \%$.

ITAs, RAs, and GSVs were cultured in the ex vivo perfusion bioreactor with DMEM supplemented with $10 \%$ FBS, $1 \%$ penicillin/streptomycin, $1 \%$ amphotericin-B, and 1\% streptomycin for $6 \mathrm{~h}$ or for 7 days under in situ (IS) or coronary-like (LAD) pulsatile media perfusion pressure and flow conditions (Table 1). For the 7 -day cultures, all media $(150 \mathrm{~mL})$ were replaced at day 4 . EC and SMC viability was confirmed at day 4 using phenylephrine $\mathrm{HCl}$ $\left(10^{-5} \mathrm{M}\right)$ and carbamylcholine chloride $\left(10^{-5} \mathrm{M}\right)$ to elicit smooth muscle-dependent contraction and endothelial-dependent relax- 
Table 1. Ex vivo culture conditions for control (in situ) and experimental (coronary-like) groups for graft tissues

\begin{tabular}{|c|c|c|c|c|c|c|}
\hline \multirow[t]{2}{*}{ Graft tissue } & \multicolumn{3}{|l|}{ In situ } & \multicolumn{3}{|c|}{ Coronary-like } \\
\hline & $\mathrm{P}, \mathrm{mm} \mathrm{Hg}$ & $\mathrm{Q}, \mathrm{mL} / \mathrm{s}$ & & $\mathrm{P}, \mathrm{mm} \mathrm{Hg}$ & $\mathrm{Q}, \mathrm{mL} / \mathrm{s}$ & \\
\hline LAD & $102 \pm 8.4$ & $0.52 \pm 0.13$ & 1.33 & $102 \pm 8.4$ & $0.52 \pm 0.13$ & 1.33 \\
\hline ITA & $100 \pm 9.5$ & $0.76 \pm 0.15$ & 1.22 & & & \\
\hline $\mathrm{RA}$ & $94 \pm 2.3$ & $0.19 \pm 0.04$ & 1.17 & & & \\
\hline GSV & $15 \pm 1.9$ & $0.17 \pm 0.03$ & 1.24 & & & \\
\hline
\end{tabular}

$\mathrm{Q}$, volumetric flow rate; $\mathrm{P}$, pressure; LAD, left anterior descending coronary artery; ITA, internal thoracic artery; RA, radial artery; GSV, great saphenous vein.

Table 2. Primer sequences of 7 remodeling-associated genes and the $A C T B$ housekeeping gene

\begin{tabular}{|c|c|c|}
\hline & & Sequence $\left(5^{\prime} \rightarrow 3^{\prime}\right)$ \\
\hline \multirow[t]{2}{*}{ COL $3 A 1$} & forward & GCGGTGACAAAGGTGAAACC \\
\hline & reverse & GGCTACCTACTGCACCTTGG \\
\hline \multirow[t]{2}{*}{ FN1 } & forward & TGGGGATACCTGGAGCAAGA \\
\hline & reverse & GCСТCTCACACTTCСАСТСС \\
\hline \multirow{2}{*}{ SERPINE1 } & forward & CCTAACCAGGCGGACTTCTC \\
\hline & reverse & CATGCGGGCTGAGACGATAA \\
\hline \multirow[t]{2}{*}{ VCAM1 } & forward & CGCTGGTCATGAATCCCGTT \\
\hline & reverse & ATCTCTGGGTCCTTGGGGAA \\
\hline \multirow[t]{2}{*}{$M M P 2$} & forward & CCTGATCTGGACCCCGAAAC \\
\hline & reverse & TATCCATCTCCGTGCTCCCA \\
\hline \multirow{2}{*}{ TGFB1 } & forward & CTAATGGTGGAAAGCGGCAA \\
\hline & reverse & $\begin{array}{l}\text { CCCGAGAGAGCAATACAG- } \\
\text { GTT }\end{array}$ \\
\hline \multirow[t]{2}{*}{ TIMP1 } & forward & CACCTGCAGTTTTGTGGCTC \\
\hline & reverse & GGGATGGATGTGCAGGGAAA \\
\hline \multirow[t]{2}{*}{$A C T B$} & forward & AGGCCAACCGTGAGAAGATG \\
\hline & reverse & CCCATCCCCAGAGTCCATGA \\
\hline
\end{tabular}

ation, respectively. At the end of each culture, sections were cut from the middle of each sample, snap-frozen on dry ice, and stored at $-80^{\circ} \mathrm{C}$ for gene expression analysis or cut and fixed in $4 \%$ paraformaldehyde for histological analysis.

Gene Expression Analysis

Total RNA was isolated from snap-frozen tissue samples using the RNeasy fibrous tissue mini kit including a homogenization step using the TissueRuptor (both from Qiagen, Hilden, Germany). Concentrations of isolated RNA were quantified using the Implen Nanophotometer Pearl (Implen, Munich, Germany). All RNA samples had $\mathrm{A}_{260} / \mathrm{A}_{280}$ and $\mathrm{A}_{260} / \mathrm{A}_{230}$ ratios $>1.9$. RNA was converted to cDNA using an iScript cDNA synthesis kit (BioRad, Hercules, CA, USA). Relative gene expression within diluted cDNA samples $\left(5 \times\right.$ in RNase-free $\left.\mathrm{H}_{2} \mathrm{O}\right)$ was then quantified via real-time PCR on a Bio-Rad CFX Connect Real-Time System us- ing Fast SYBR Green Master Mix (Applied Biosystems, Foster City, CA, USA). Seven genes associated with vascular remodeling were selected for analysis as mentioned, and $\beta$-actin $(A C T B)$ was used as a housekeeping gene (Table 2). Samples $(n=3)$ were run in triplicate, and all plate configurations were run 3 times. Fluorescence threshold (300 RFU) was set the same for each plate to enable comparison across different plates. Relative gene expression was calculated using the Pfaffl method [29]. Melt curve analysis was performed for each real-time PCR run.

\section{Histological Analysis}

Paraformaldehyde-fixed samples were embedded in paraffin, sectioned with a microtome at $5-\mu \mathrm{m}$ thick, and stained with hematoxylin/eosin (H\&E). Digital images of stained slides were acquired on a Nikon E600 microscope (Nikon Instruments, Melville, NY, USA) using QCapture imaging software (QImaging, Surrey, BC, Canada).

\section{Protein Expression Analysis}

Serial sections of paraffin-embedded representative vessels were stained for MMP2, tissue inhibitor of metallopeptidase 1 (TIMP1), and plasminogen activator inhibitor type 1 (PAI1) proteins. Rabbit polyclonal anti-MMP2 (ab97779), rabbit polyclonal anti-TIMP1 (ab231485), and rabbit polyclonal anti-PAI1 (ab66705) antibodies were obtained from Abcam, and stained using a 3,3'-diaminobenzidinetrahydrochloride (DAB) immuneperoxidase kit (Dako, Carpinteria, CA, USA). All slides were stained in one batch for each antibody to reduce variation. Digital images of stained slides were acquired on a Nikon E600 microscope using QCapture software.

Semiquantitative analysis of protein expression was determined by counting the number of brown (i.e., positively stained) pixels relative to total tissue area in each sample for each protein. Using ImageJ software (National Institutes of Health), a region of interest was drawn manually on each sample to include a representative region of the vessel wall, and the area fraction of brown pixels to total tissue area was quantified using the "Threshold_Colour" plugin (hue: bandstop 40-215, saturation: bandpass 19-255, and brightness: bandpass 0-209) [30]. Cultured samples (7-day IS and LAD) were compared to noncultured samples ( $\mathrm{t} 0$ ) to evaluate the level of protein expression resulting from ex vivo culture and correlate with gene expression studies. 
Fig. 2. Comparison of remodeling-associated gene expression of freshly isolated (time point zero, t0) LAD, ITA, RA, and GSV. ${ }^{*} p<0.05$ with respect to LAD; ${ }^{*} p<$ 0.05 with respect to ITA (2-tailed, 2 -sample Student's $t$ test; $n=6)$. No genes were significantly different in the RA and GSV samples.
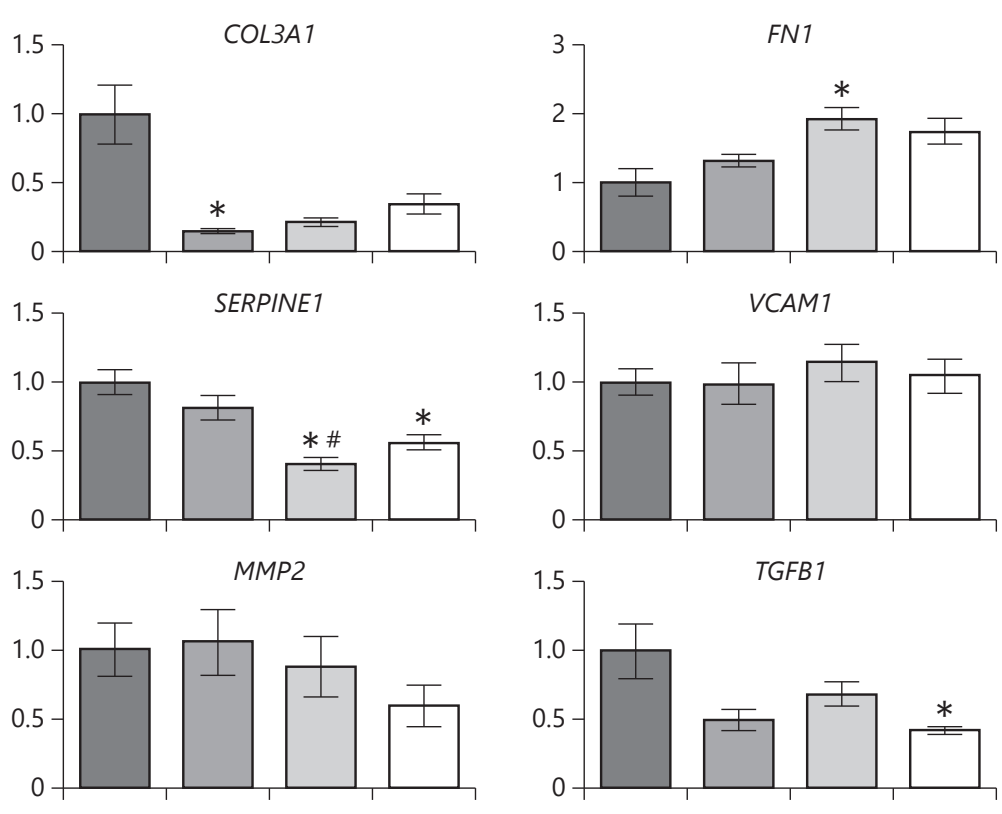

MMP2
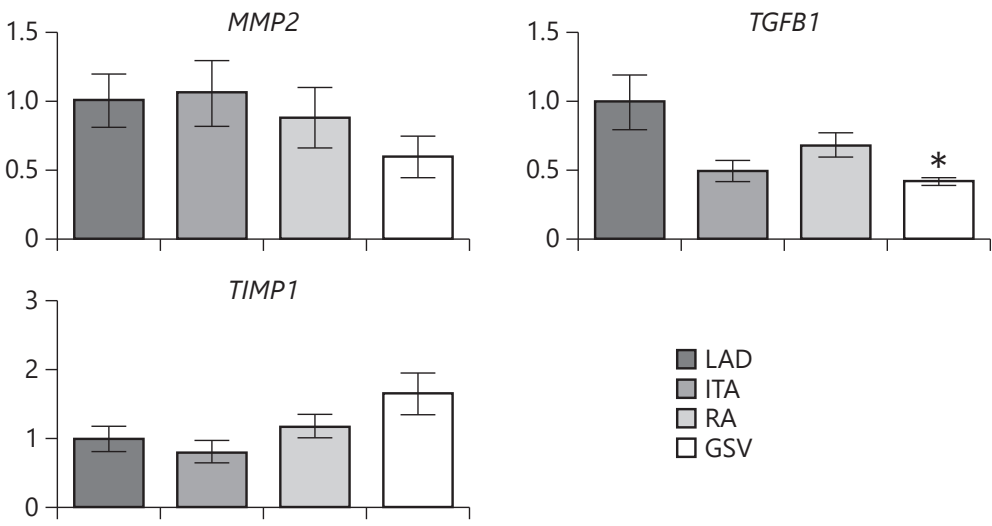

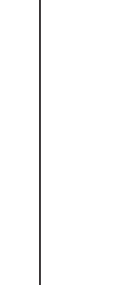$$
\text { . }
$$

\section{Statistical Analysis}

Data are reported as the mean \pm SEM of all measurements for a given experimental group. All cultured groups had a sample size $n=3$, and at t $0 n=6$. Two-tailed, 2 -sample, Student's $t$ tests assuming unequal variance were conducted at $\alpha=0.05$ between reference samples (see "Gene Expression Analysis" above) and experimental groups.

\section{Results}

\section{Basal Gene Expression}

To provide a baseline comparison of healthy gene expression, the genes associated with remodeling were compared between freshly isolated LAD, ITA, RA, and GSV samples $(n=6)$ immediately after sacrificing the animals (Fig. 2). Overall, basal gene activity was similar in all 4 vessel types, with certain notable differences. COL3A1 $(p=0.030$ ) was the only gene found to be statistically lower when comparing the basal gene expression in the ITA and LAD. Basal gene expression of FN1 ( $p=$ 0.049) however, was found to be significantly higher in the RA than in the LAD. SERPINE1 was lower in the RA than in the LAD $(p=0.002)$ and ITA $(p=0.031)$. SERPINE1 $(p=0.021)$ and TGFB1 $(p<0.001)$ were both lower in the GSV than in the LAD.

\section{Comparison of Primary Vascular Cells}

Cell populations were successfully isolated from the intimal, medial, and adventitial layers of the LAD, ITA, RA, and GSV samples. Confocal microscopy qualitatively suggested intimal populations consisting of mostly EC, medial populations consisting of mostly SMC, and adventitial populations consisting of mostly fibroblasts. Intimal populations appeared qualitatively similar across vessel types and with characteristics similar to disorganized EC (Fig. 3). Medial populations appeared to consist primarily of spindle-shaped SMCs in the ITA samples, but the LAD, RA, and GSV samples appeared to have significant populations of non-spindle-shaped cells as well. Initial adventitial population sizes varied widely across vessels due to the amount of tissue available and the perivascular environment of each vessel. Once in culture, cells 


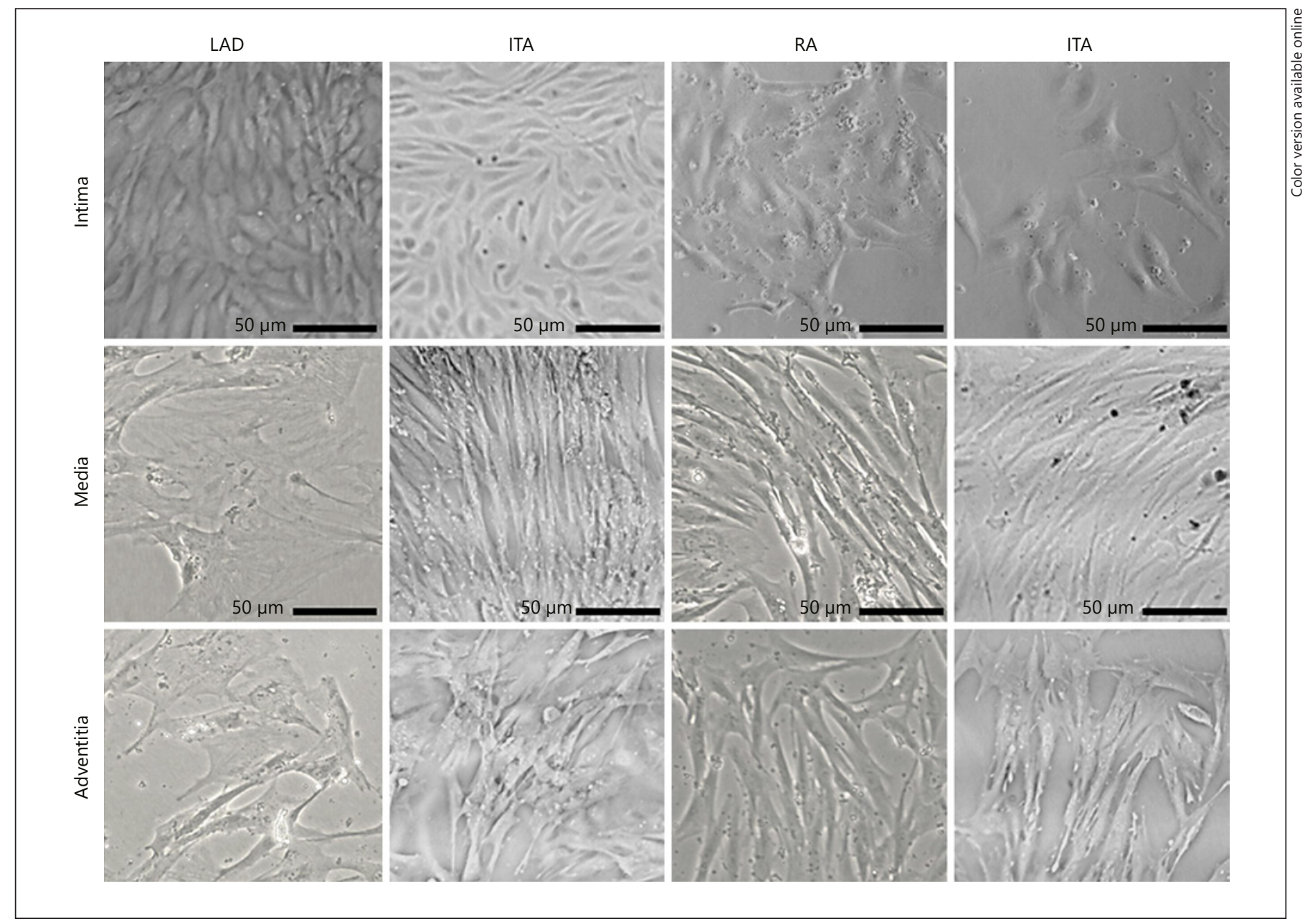

Fig. 3. Representative phase contrast images of isolated cells harvested from the intimal, medial, and adventitial layers of the LAD, ITA, RA, and GSV after 7 days in culture plates. $\times 20$.

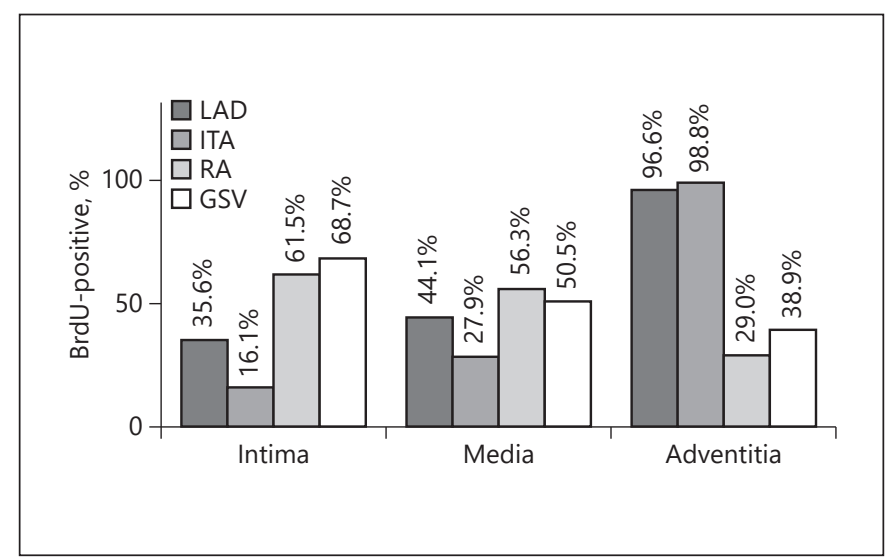

Fig. 4. BrdU-positive staining of isolated cells harvested from the intimal, medial, and adventitial layers of the LAD, ITA, RA, and GSV after 7 days indicates the \% of proliferating cells. from all vessels appeared similar, exhibiting classic stellate fibroblast morphology.

BrdU uptake suggested variable proliferation rates across both layers and vessel types at 7 days (Fig. 4). RA and GSV intimal populations proliferated (131/213 and $46 / 67$ observed cells, respectively) at rates higher than in the LAD and ITA (141/396 and 66/410, respectively). Proliferation rates of medial cells appeared relatively similar in the LAD, RA, and GSV samples observed (89/202, $27 / 48$, and $108 / 214$, respectively), but the ITA samples exhibited a lower proportion of proliferating cells (60/215). In the adventitial samples observed, almost all the cells in the LAD and ITA exhibited BrdU uptake (28/29 and 85/86, respectively), but the RA and GSV cells had much lower proportions (18/62 and 44/113, respectively). 
Fig. 5. Comparison of remodeling-associated gene expression changes in ITA, RA, and GSV samples as a result of acute $(6 \mathrm{~h})$ and prolonged (7 days) ex vivo tissue culture exposed to media perfusion approximating in situ (IS) or LAD conditions. ${ }^{*} p<0.05$ with respect to 6 -h IS; ${ }^{\#} p<0.05$ with respect to 6-h LAD (2-tailed, 2-sample Student's $t$ test; $n=3)$.
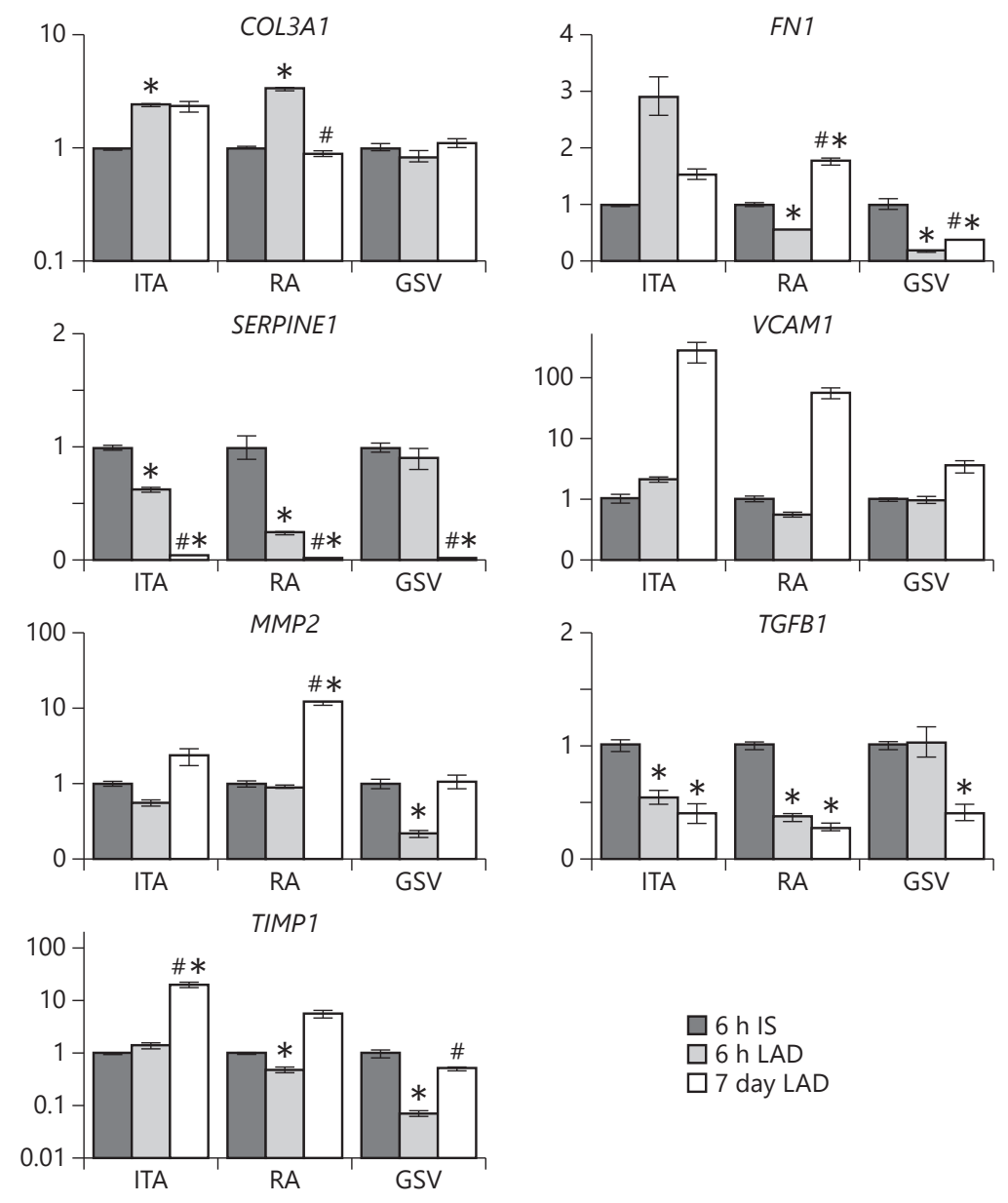

\section{Effects of CABG Loading Conditions on Gene}

\section{Expression}

To assess the early remodeling effects of the mechanical stimuli characteristic of the coronary environment, gene expression activity was compared between ITAs, RAs, and GSV s cultured under IS or LAD conditions and measured after $6 \mathrm{~h}$ and 7 days of perfusion culture (Fig. 5). Collectively, $66.7 \pm 9.91 \%$ of the genes tested after $6 \mathrm{~h}$ and $52.4 \pm 7.27 \%$ of those tested after 7 days were disturbed by IS culture when compared to acutely measured gene expression.

In total, 4 ITA genes were affected by the short- and long-term LAD culture conditions versus the IS culture conditions. The ITA had significantly higher COL $3 A 1$ expression $(p<0.001)$ and significantly lower SERPINE1 ( $p$ $=0.004)$ and TGFB1 $(p=0.025)$ expression at $6 \mathrm{~h}$ of LAD culture. SERPINE1 $(p<0.001)$ and TGFB1 $(p=0.021)$ ex- pression remained low after 7 days of LAD culture, but a significant increase in TIMP1 $(p=0.027)$ expression occurred.

A total of 6 RA genes were affected by the short- and long-term LAD culture conditions. The RA had significantly higher COL $3 A 1$ expression $(p<0.001)$ but lower FN1 $(p<0.001)$, SERPINE1 $(p=0.016)$, TGFB1 $(p<$ $0.001)$, and TIMP1 $(p=0.004)$ expression at $6 \mathrm{~h}$ of LAD culture. After 7 days of LAD culture, COL3A1 returned to basal levels; SERPINE1 $(p=0.005)$ and TGFB1 $(p<$ $0.001)$ decreased further, and significant increases in FN1 $(p<0.001)$ and MMP2 $(p=0.006)$ expression were observed.

Five GSV genes in total were affected by LAD culture conditions compared to the IS conditions. FN1 ( $p=$ $0.005), M M P 2(p=0.035)$, and TIMP1 $(p=0.033)$ were all decreased at $6 \mathrm{~h}$ of LAD conditions compared to $6 \mathrm{~h}$ 

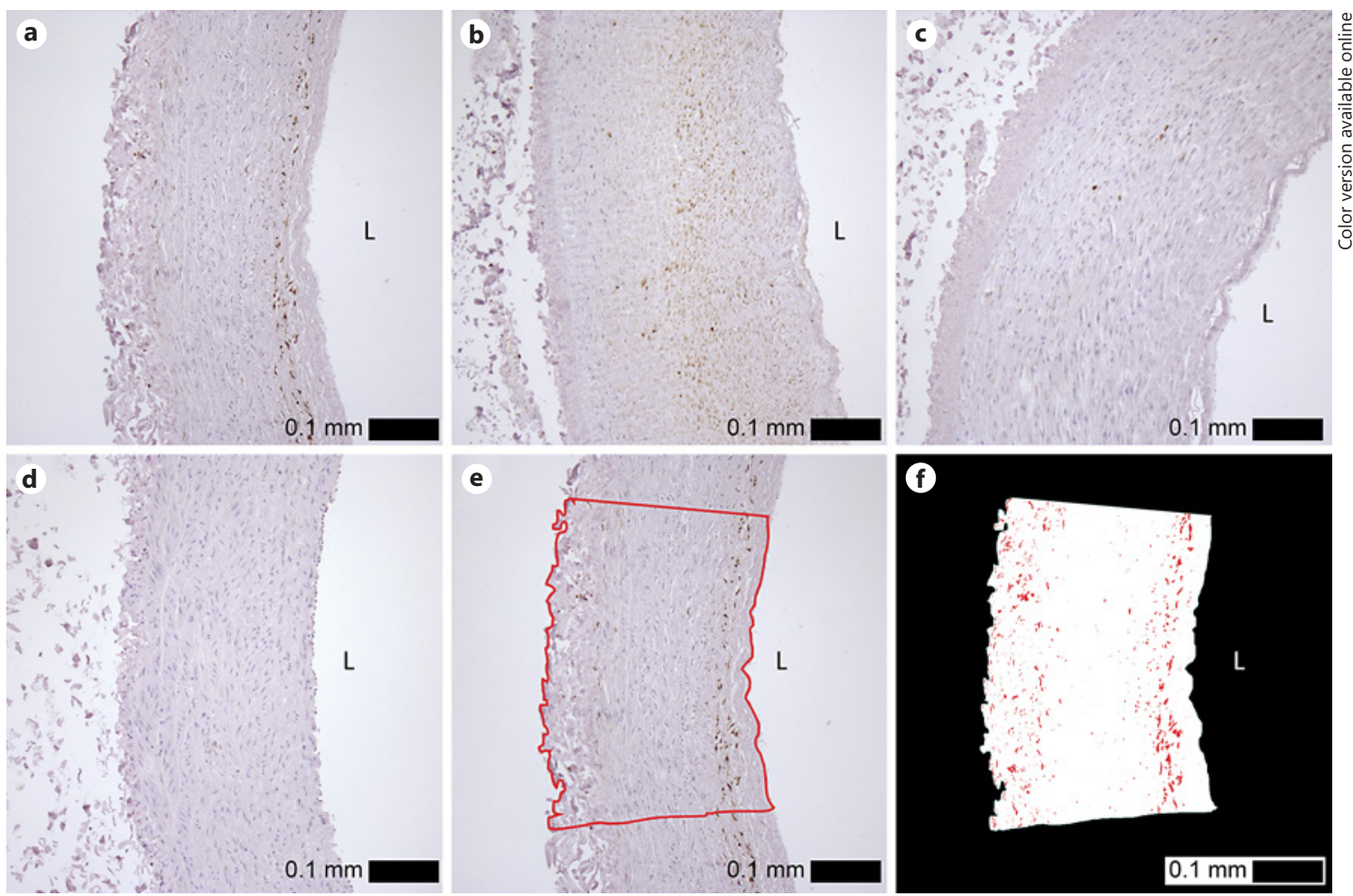

e
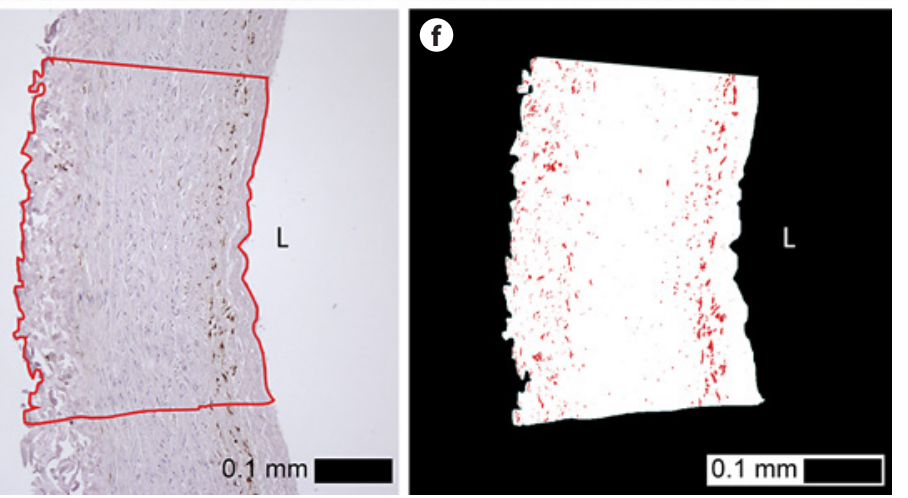

Fig. 6. Representative images of protein expression analyzed via DAB staining of anti-PAI1 antibody after 7 days of LAD culture in the ITA (a), RA (b), and GSV (c) as well as an image showing minimal PAI1 expression in a noncultured ITA (d). e, $\mathbf{f}$ The thresholding protocol used to quantify the area fraction occupied by positively stained pixels on the same image as a. The lumen side of each vessel is indicated with $\mathrm{L}$.

IS. After 7 days of LAD culture, FN1 $(p=0.020)$ gene expression actually increased compared to at $6 \mathrm{~h}$, but was still less than at $6 \mathrm{~h}$ IS $(p=0.001)$.

\section{Effect of CABG Loading Conditions on Protein Expression}

Three proteins corresponding to genes assessed via qPCR, i.e., PAI1, MMP2, and TIMP1, were analyzed semiquantitatively via $\mathrm{DAB}$ staining and image analysis to assess the levels of protein expression before and after ex vivo culture and to correlate gene expression findings (Fig. 6, 7).

Qualitatively, positively stained regions (brown) showed PAI1 expression in the 7-day IS and LAD samples in the ITA, RA, and GSV (Fig. 6a-c); t0 samples (i.e., before culture) did not indicate PAI1 expression (Fig. 6d). Positive regions of PAI1 staining extended throughout the intima, media, and adventitia, but localization varied across samples. Similarly, RA, and GSV vessels stained for TIMP1 had distinct regions of positive staining, whereas t0 and ITA samples suggested little-to-no TIMP1 protein expression. In TIMP1-stained RAs, staining was primarily localized in the intima and inner half of the media. In TIMP1-stained GSVs, staining was primarily localized in the outer half of the media. Across culture conditions and vessel types, samples stained for MMP2 showed little apparent positive staining.

Positively stained regions were quantified by image analysis as a percentage of total tissue area (Fig. 7), and the quantified protein expression was aligned with the qualitative observations. For PAI1, the day-7 LAD ITA ( $p=$ $0.023)$ and IS RA $(p=0.040)$ samples showed significant increases in positively stained pixels compared to their respective t0 counterparts. For MMP2, the day-7 IS ITA sample $(p=0.002)$ showed a significant increase in stained pixels compared to t0, and the day-7 LAD GSV sample $(p=0.009)$ showed a significant decrease relative to t0. For TIMP1, all day-7 samples showed an increase in positive staining that was statistically insignificant versus t 0 , likely due to the relatively large variation between samples. 


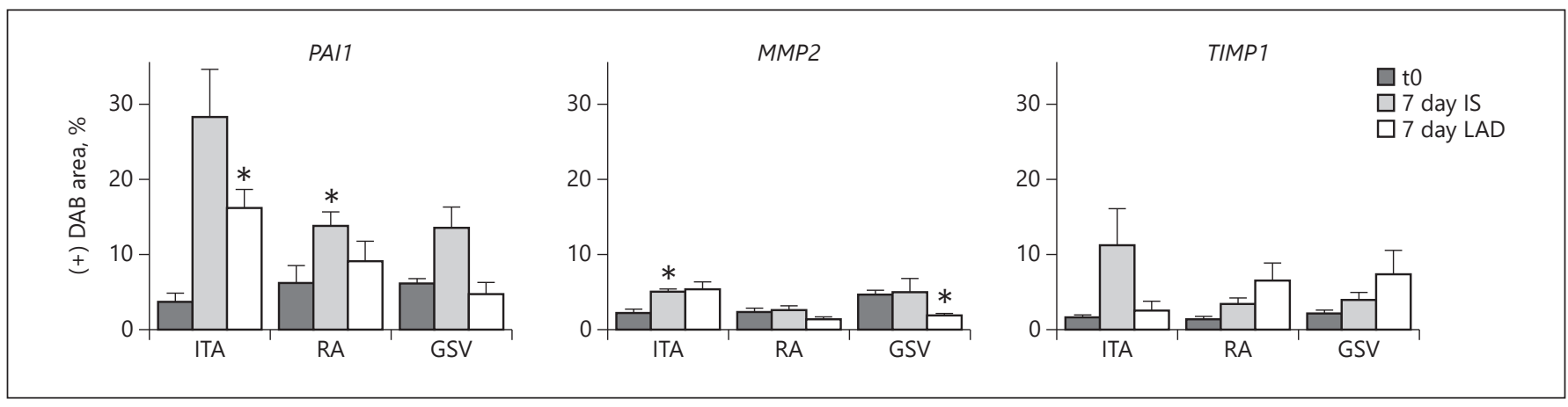

Fig. 7. Relative protein expression of PAI1, MMP2, and TIMP1 as quantified by area fraction of pixels positively stained via immunohistochemistry. Error bars represent mean \pm SEM of $n=6(\mathrm{t} 0)$ or $n=3$ (day 7 IS and LAD) samples. ${ }^{*} p<0.05$ with respect to t0.

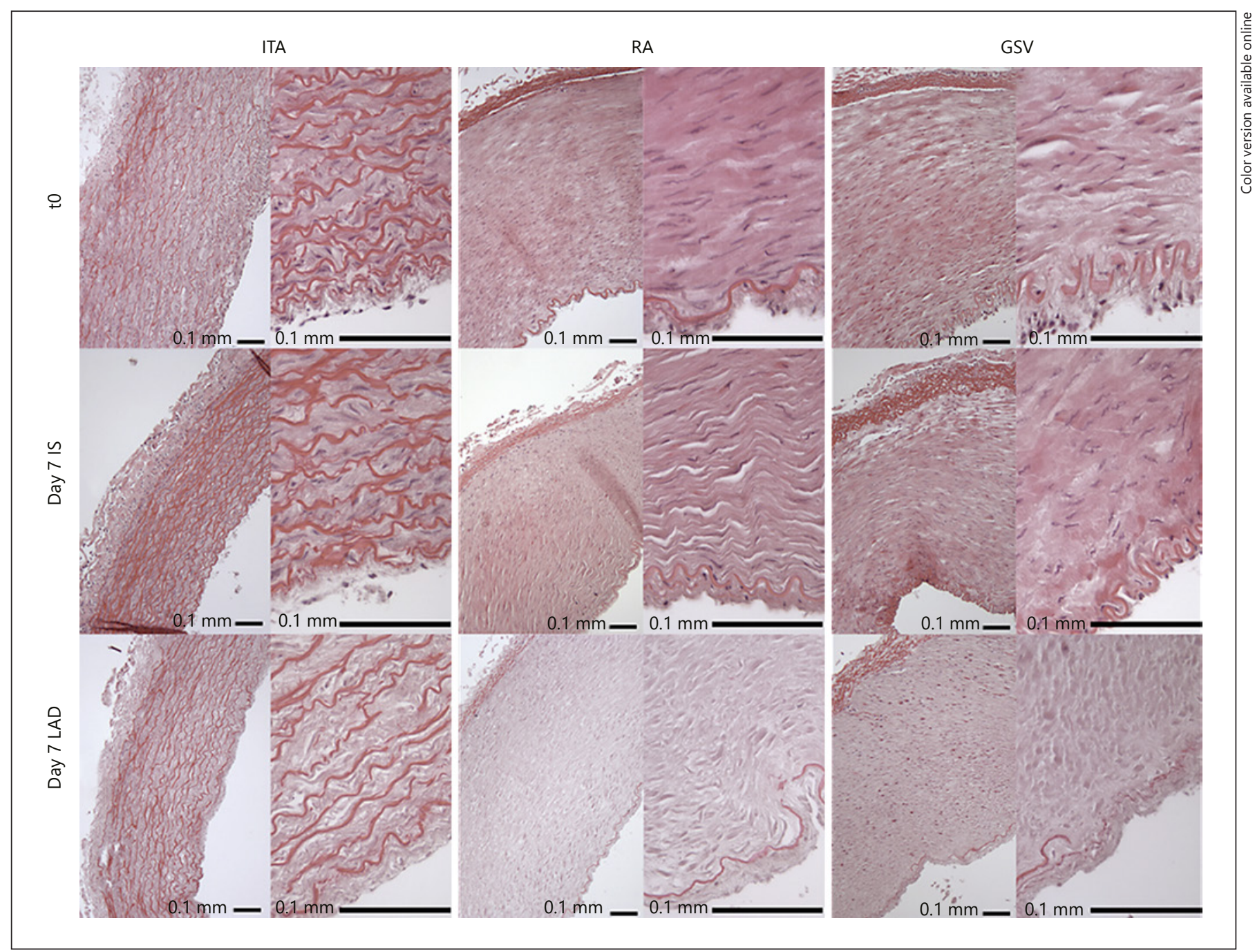

Fig. 8. Representative H\&E-stained cross-sections of the ITA, RA, and GSV before (top) and after (middle; bottom) 7 days of ex vivo perfusion culture in in situ (IS; middle row) or left anterior descending coronary artery (LAD; bottom row) culture conditions. All images are paired at $\times 100$ (left) and $\times 400$ (right). Scale bar, $0.1 \mathrm{~mm}$. 
Effect of ex vivo Vessel Culture on Histology

ITAs, RAs, and GSVs at day 0 were histologically distinct (Fig. 8, top). Most notable was the presence of approximately 15 elastic lamellae in the ITA samples and a thinner medial layer that was obvious even on an H\&E stain. In contrast, RAs and GSVs contained a thick, muscular media with well-organized cell nuclei, often folding in the histological sections. In the RAs and GSVs, a single internal elastic lamina was prominent. Despite differences in gene expression, IS-cultured vessels were morphologically similar when comparing between the acute and 7day time points for any given vessel (Fig. 8, middle). The only notable difference was an apparent decrease in EC continuity. Overall, the ITA histology appeared insensitive to IS versus LAD culture conditions. RAs and GSVs cultured under LAD conditions for 7 days, however, showed marked changes in cell and tissue morphology compared to at $t 0$ or at 7 days of IS. The endothelial layers showed sparse nuclei and a gradient in medial cells was apparent, with the innermost cells being necrotic or pyknotic and the outermost cells appearing otherwise healthy.

\section{Discussion}

This investigation sought to understand the early remodeling processes occurring in CABG tissues after implantation. We used pulsatile tissue perfusion as a tool to evaluate whether the 3 most common CABG tissues have inherently different abilities to undergo adaptive remodeling. To this end, primary vascular cells were isolated from LAD, ITA, RA, and GSV samples, and an ex vivo pulsatile perfusion bioreactor was utilized to culture porcine samples under IS pressure and flow conditions, and conditions representative of the coronary vasculature. Cell cultures demonstrated variable rates of mitotic activity across vessel types and layers. The 6-h cultures were utilized to elucidate the acute response of graft tissues after CABG, and the 7-day cultures were utilized to elucidate remodeling responses as vessels started to adapt to chronic mechanical changes. The expression of 7 genes associated with vascular remodeling were evaluated as a proxy for activated remodeling pathways and downstream remodeling outcomes, and protein expression of 3 genes confirmed the validity of gene expression data, while histological staining provided qualitative evidence of morphological changes due to ex vivo culture conditions.

Our previous work quantified the homeostatic mechanics of the LAD, ITA, RA, and GSV in an IS loading environment and demonstrated that the amount of mechanical de- viation caused by implantation in the coronary circulation was different for the candidate graft vessels [31]. Several other study groups have published investigations involving ex vivo culture relating to CABG $[9,23,32-36]$. These tissue-level culture studies allow for specific mechanical loading conditions and natural interactions between cell types that are not possible in flat-plate culture studies. Most tissue cultures studies, however, have focused almost exclusively on saphenous veins due to the implication of vein grafts in many CABG failures, and each investigation has focused on different aspects of graft remodeling: viability/ apoptosis [35], shear stress versus pressure [9], cell proliferation [33], morphometry [37], and oxygenation [34]. However, none has focused on the differential remodeling ability of ITAs, RAs, and GSVs. Whereas GSV s have an historical precedence in CABG grafting, ITAs are the standard-of-care, and RAs constitute an option for second and third bypass graft procedures [38].

The degree towards which coronary-like culture conditions drive pathological remodeling in both the RA and GSV was surprising. Specifically, RAs and GSVs (and to a lesser extent, ITAs), when exposed to coronary-culture conditions, but not to IS conditions, experience a considerable loss of endothelium and limited apoptosis of cells in the inner layer of the media. These vessels would likely reendothelialize rapidly in vivo and repopulate with mural cells [39]; however, such processes could be the difference between healthy and pathological remodeling.

A functioning endothelium plays a critical role in SMC phenotype modulation, and the denudation process is known to cause intimal hyperplasia, adventitial cell proliferation, fibrosis, and constrictive remodeling [40]. Our work supports the theory that many cells of transplanted tissues are lost soon after transplantation and repopulated with host-derived cells $[39,41,42]$. In contrast, the outer layers of the media retained a normal looking histology, remarkably similar to in vessels prior to culture. This observation has important implications, with adventitial fibroblasts and progenitor cells playing critical roles in the remodeling of vascular grafts [43].

We anticipated that ITAs, RAs, and GSVs exposed to LAD pressure and flow conditions would experience an acute, pathological deviation in the expression of many of the genes when compared to their matched IS culture conditions, followed by an eventual restoration of basal levels as the tissues restored homeostasis. This effect was observed for COL3A1 and FN1 genes in all vessels, and for MMP2 (in ITA and GSV only) and TIMP1 (in RA and GSV only) to a lesser extent. In contrast, SERPINE1 and $T G F B 1$ continued a path of decreased expression at 7 days 
whereas VCAM1 only increased. Gene expression results were confirmed through protein expression analysis of PAI1, MMP2, and TIMP1, which showed qualitative and quantitative changes after 7 days of culture under IS and LAD culture conditions. Protein expression generally agreed with gene expression patterns for the 3 genes studied, especially PAI1, which has been highlighted by other authors for its elevated expression in the remodeling response to CABG $[9,44]$.

Such findings suggest that homeostasis was still not observed at this time point, and such behavior is consistent with previous in vivo remodeling studies that indicated that 2-3 weeks are necessary for gross changes in tissue structure [13]. VCAM1 and SERPINE1 are both genes expressed predominantly by EC, and so pathological conditions are likely, given the scarcity of EC in the cultures of RAs and GSVs under LAD flow conditions. Despite this, sufficient concentrations of RNA were procured from pre- and postculture cell populations for data analysis. Comparisons between IS and LAD cultures of RA and GSVs also support the approach that long-term ex vivo conditioning in a bioreactor should use a stepwise or ramping approach to transition from IS to coronarylike media dynamics $[45,46]$. This approach has been shown to generate stable solutions and prevent maladaptation in computational simulations of vein remodeling [47]. Furthermore, whereas this investigation utilized simple sinusoid arterial flow and pressure waveforms, future investigations should evaluate ex vivo remodeling in a bioreactor where flow, pressure, and the phase angle between them are accurately replicated in order to most closely resemble physiological CABG conditions [48].

For the most part, gene expression profiles were similar across vessels when measured immediately after sacrifice, with COL3A1 found to be the only gene differentially expressed by the ITA compared to the LAD while, SERPINE1, FN1 (RA only), and TGFB1 (GSV only) were found to be different in the RA and GSV when compared to the LAD. These striking findings provide important information regarding basal gene expression from different tissue sources irrespective of tissue culture. We further expected that, because the RA and GSV experience increased levels of pressure and flow in LAD culture, they would result in similarly regulated gene profiles. Such behavior, however, was observed for most genes except COL3A1. In contrast, the ITA IS conditions were most like those of the LAD, so it was supportive to observe modest changes in gene expression at $6 \mathrm{~h}$ of LAD culture for this tissue.

In theory, this experimental design should allow for a clear identification of early remodeling pathways stimu-

Perfusion Tissue Culture and Remodeling of ITA, RA, and GSV lated by $\mathrm{CABG}$, as the bioreactor was designed to perfuse vascular tissue with pressure and flow levels equivalent to the coronary circulation or any other desired loading conditions. However, vessels were unavoidably exposed to random variables, which could confound the results of the investigation. To account for these variables, control cultures were performed under IS loading conditions for each vessel. If environmental stimuli remained unchanged for these control groups compared to their basal IS state (i.e., the t0 results), then we would not have seen differences in gene expression or histology for these groups. Unsurprisingly, this was not the case, with the expression of $52.4 \pm 7.27 \%$ of the genes being different after 7 days of IS culture conditions compared to their basal (freshly harvested) expression levels. This could be due to a number of factors: the substitution of blood for culture medium, a lack of perivascular tissue, an incomplete inflammatory response, or insufficient replication of physiological hemodynamic waveforms. As it stands, attention should be focused on the differential responses as a result of culture under coronary-like versus IS loading conditions rather than with reference to the freshly harvested tissues.

The results of this investigation indicate that primary intimal, medial, and adventitial cells from selected source tissues exhibit similar morphology but vary in their rates of proliferation when isolated and cultured under the same conditions for 7 days. When tissues were cultured in an ex vivo perfusion bioreactor, molecular pathways associated with vascular remodeling were activated as early as $6 \mathrm{~h}$ after implantation in a new environment, and these pathways were sensitive to the specific medial perfusion dynamics to which the vessel was exposed. Some effects appear to be, at least partially, the result of ex vivo culture, but responses occurring differentially between IS and LAD cultures were the result of chronic deviation from homeostasis.

Moreover, some, but not all, of these remodeling-associated pathways were differentially activated across our 3 graft vessel types, which, together with differing observed rates of cell proliferation, suggests the possibility of differential propensity for vascular remodeling and adaptation when used for CABG. It was therefore intuitive that significantly different outcomes would occur for these vessels when used clinically for CABG. In situ, each vessel has a unique homeostatic state of stress, and all vessels experience different changes in loading due to grafting [31]. Thus, using a continuum mechanics framework and prolonged tissue culture ( 3 weeks), our future investigations will seek to isolate the inherent remodeling capacity of potential graft vessels from the mechanical deviation to which they have been subjected. 


\section{Acknowledgements}

The authors would like to acknowledge the experimental contributions of summer medical student Nicole Carey to the current work. This work was supported by the National Institutes of Health (NIH) P20GM103444 and R21EB022131 grants.

\section{Disclosure Statement}

The authors report no conflicts of interest relating to this manuscript.

\section{References}

1 Carey JS, Danielsen B, Milliken J, Li Z, Stabile BE. Narrowing the gap: early and intermediate outcomes after percutaneous coronary intervention and coronary artery bypass graft procedures in California, 1997 to 2006. J Thorac Cardiovasc Surg. 2009 Nov;138(5):11007.

2 Benjamin EJ, Blaha MJ, Chiuve SE, Cushman M, Das SR, Deo R, et al. Heart Disease and Stroke Statistics - 2017 Update: A Report from the American Heart Association. Circulation 2017 Mar;135(10):e146-e603.

3 Harskamp RE, Lopes RD, Baisden CE, de Winter RJ, Alexander JH. Saphenous vein graft failure after coronary artery bypass surgery: pathophysiology, management, and future directions. Ann Surg. 2013 May;257(5): 824-33.

4 Davies MG, Hagen PO. Reprinted article "Pathophysiology of vein graft failure: a review". Eur J Vasc Endovasc Surg. 2011 Sep;42 Suppl 1:S19-29.

5 Owens CD, Gasper WJ, Rahman AS, Conte MS. Vein graft failure. J Vasc Surg. 2015 Jan; 61(1):203-16.

6 Fung YC. What are the residual stresses doing in our blood vessels? Ann Biomed Eng. 1991; 19(3):237-49.

7 Rachev A, Greenwald S, Shazly T. Are geometrical and structural variations along the length of the aorta governed by a principle of "optimal mechanical operation"? J Biomech Eng. 2013 Aug;135(8):81006.

8 Jones EA, le Noble F, Eichmann A. What determines blood vessel structure? Genetic prespecification vs. hemodynamics. Physiology (Bethesda). 2006 Dec;21(6):388-95.

9 Berard X, Déglise S, Alonso F, Saucy F, Meda $\mathrm{P}$, Bordenave $\mathrm{L}$, et al. Role of hemodynamic forces in the ex vivo arterialization of human saphenous veins. J Vasc Surg. 2013 May; 57(5):1371-82.

10 Kudo FA, Muto A, Maloney SP, Pimiento JM, Bergaya S, Fitzgerald TN, et al. Venous identity is lost but arterial identity is not gained during vein graft adaptation. Arterioscler Thromb Vasc Biol. 2007 Jul;27(7):1562-71.

11 Eberth JF, Gresham VC, Reddy AK, Popovic $\mathrm{N}$, Wilson E, Humphrey JD. Importance of pulsatility in hypertensive carotid artery growth and remodeling. J Hypertens. 2009 Oct;27(10):2010-21.
12 Eberth JF, Cardamone L, Humphrey JD. Evolving biaxial mechanical properties of mouse carotid arteries in hypertension. J Biomech. 2011 Sep;44(14):2532-7.

13 Eberth JF, Popovic N, Gresham VC, Wilson E, Humphrey JD. Time course of carotid artery growth and remodeling in response to altered pulsatility. Am J Physiol Heart Circ Physiol. 2010 Dec;299(6):H1875-83.

14 Chiu JJ, Chien S. Effects of disturbed flow on vascular endothelium: pathophysiological basis and clinical perspectives. Physiol Rev. 2011 Jan;91(1):327-87.

15 Chiu JJ, Usami S, Chien S. Vascular endothelial responses to altered shear stress: pathologic implications for atherosclerosis. Ann Med. 2009;41(1):19-28.

16 Haga JH, Li YS, Chien S. Molecular basis of the effects of mechanical stretch on vascular smooth muscle cells. J Biomech. 2007;40(5): 947-60.

17 Humphrey JD, Eberth JF, Dye WW, Gleason RL. Fundamental role of axial stress in compensatory adaptations by arteries. J Biomech. 2009 Jan;42(1):1-8.

18 Wilson E, Mai Q, Sudhir K, Weiss RH, Ives HE. Mechanical strain induces growth of vascular smooth muscle cells via autocrine action of PDGF. J Cell Biol. 1993 Nov;123(3):741-7.

19 Majesky MW. Developmental basis of vascular smooth muscle diversity. Arterioscler Thromb Vasc Biol. 2007 Jun;27(6):1248-58.

20 Awgulewitsch A, Majesky MW. Interpreting inflammation: smooth muscle positional identity and nuclear factor- $\mathrm{kB}$ signaling. Arterioscler Thromb Vasc Biol. 2013 Jun;33(6): 1113-5.

21 Intengan HD, Schiffrin EL. Vascular remodeling in hypertension: roles of apoptosis, inflammation, and fibrosis. Hypertension. 2001 Sep;38(3 Pt 2):581-7.

22 Galis ZS, Khatri JJ. Matrix metalloproteinases in vascular remodeling and atherogenesis: the good, the bad, and the ugly. Circ Res. 2002 Feb;90(3):251-62.

23 Dummler S, Eichhorn S, Tesche C, Schreiber U, Voss B, Deutsch MA, et al. Pulsatile ex vivo perfusion of human saphenous vein grafts under controlled pressure conditions increases MMP-2 expression. Biomed Eng Online. $2011 \mathrm{Jul} ; 10(1): 62$.
24 Tamura K, Chen YE, Lopez-Ilasaca M, Daviet L, Tamura N, Ishigami T, et al. Molecular mechanism of fibronectin gene activation by cyclic stretch in vascular smooth muscle cells. J Biol Chem. 2000 Nov;275(44):34619-27.

25 Feng Y, Yang JH, Huang H, Kennedy SP, Turi TG, Thompson JF, et al. Transcriptional profile of mechanically induced genes in human vascular smooth muscle cells. Circ Res. 1999 Dec;85(12):1118-23.

26 Sorescu GP, Sykes M, Weiss D, Platt MO, Saha A, Hwang J, et al. Bone morphogenic protein 4 produced in endothelial cells by oscillatory shear stress stimulates an inflammatory response. J Biol Chem. 2003 Aug;278(33): 31128-35.

27 O'Callaghan CJ, Williams B. Mechanical strain-induced extracellular matrix production by human vascular smooth muscle cells: role of TGF-beta(1). Hypertension. 2000 Sep; 36(3):319-24.

28 Azhar M, Schultz JJ, Grupp I, Dorn GW 2nd, Meneton P, Molin DG, et al. Transforming growth factor beta in cardiovascular development and function. Cytokine Growth Factor Rev. 2003 Oct;14(5):391-407.

29 Pfaffl MW. A new mathematical model for relative quantification in real-time RT-PCR. Nucleic Acids Res. 2001 May;29(9):e45-45.

30 Landini G. Advanced shape analysis with ImageJ. In: Proceedings of the Second ImageJ User and Developer Conference; 2008; Luxembourg. p. 116-121.

31 Prim DA, Zhou B, Hartstone-Rose A, Uline MJ, Shazly T, Eberth JF. A mechanical argument for the differential performance of coronary artery grafts. J Mech Behav Biomed Mater. 2016 Feb;54:93-105.

32 Déglise S, Martin D, Probst H, Saucy F, Hayoz $\mathrm{D}$, Waeber $\mathrm{G}$, et al. Increased connexin 43 expression in human saphenous veins in culture is associated with intimal hyperplasia. J Vasc Surg. 2005 Jun;41(6):1043-52.

33 Voisard R, Ramiz E, Baur R, GastrockBalitsch I, Siebeneich H, Frank O, et al. Pulsed perfusion in a venous human organ culture model with a Windkessel function (pulsed perfusion venous HOC-model). Med Sci Monit. 2010 Nov;16(11):CR523-9.

34 Piola M, Prandi F, Fiore GB, Agrifoglio M, Polvani G, Pesce M, et al. Human Saphenous Vein Response to Trans-wall Oxygen Gradients in a Novel Ex Vivo Conditioning Platform. Ann Biomed Eng. 2016 May;44(5):1449-61. 
35 Miyakawa AA, Dallan LAO, Lacchini S, Borin TF, Krieger JE. Human saphenous vein organ culture under controlled hemodynamic conditions. Clinics. 2008 Oct;63(5):683-8.

36 Campos LC, Miyakawa AA, Barauna VG, Cardoso L, Borin TF, Dallan LA, et al. Induction of CRP3/MLP expression during vein arterialization is dependent on stretch rather than shear stress. Cardiovasc Res. 2009 Jul; 83(1):140-7.

37 Piola M, Prandi F, Bono N, Soncini M, Penza E, Agrifoglio M, et al. A compact and automated ex vivo vessel culture system for the pulsatile pressure conditioning of human saphenous veins. J Tissue Eng Regen Med. 2016 Mar;10(3):E204-15.

38 Loop FD, Lytle BW, Cosgrove DM, Stewart RW, Goormastic M, Williams GW, et al. Influence of the internal-mammary-artery graft on 10-year survival and other cardiac events. N Engl J Med. 1986 Jan;314(1):1-6.
$39 \mathrm{Hu}$ Y, Davison F, Zhang Z, Xu Q. Endothelial replacement and angiogenesis in arteriosclerotic lesions of allografts are contributed by circulating progenitor cells. Circulation. 2003; 108(25):3122-7.

40 Lindner V. Balloon Denudation of Blood Vessels. In: Augustin HG, editor. Methods in Endothelial Cell Biology. Berlin, Heidelberg: Springer; 2004. p. 187-95.

$41 \mathrm{Hu}$ Y, Mayr M, Metzler B, Erdel M, Davison $\mathrm{F}, \mathrm{Xu} \mathrm{Q}$. Both donor and recipient origins of smooth muscle cells in vein graft atherosclerotic lesions. Circ Res. 2002; Oct;91(7):e1320.

42 Zhang L, Freedman NJ, Brian L, Peppel K. Graft-extrinsic cells predominate in vein graft arterialization. Arterioscler Thromb Vasc Biol. 2004;24(3):470-476.

43 Leach DF, Nagarkatti M, Nagarkatti P, Cui T. Functional states of resident vascular stem cells and vascular remodeling. Front Biol (Beijing). 2015 Oct;10(5):387-97.
44 Saucy F, Probst H, Alonso F, Bérard X, Déglise S, Dunoyer-Geindre S, et al. Ex vivo pulsatile perfusion of human saphenous veins induces intimal hyperplasia and increased levels of the plasminogen activator inhibitor 1. Eur Surg Res. 2010;45(1):50-9.

45 Gusic RJ, Petko M, Myung R, William Gaynor J, Gooch KJ. Mechanical properties of native and ex vivo remodeled porcine saphenous veins. J Biomech. 2005 Sep;38(9):1770-9.

46 Gusic RJ, Myung R, Petko M, Gaynor JW, Gooch KJ. Shear stress and pressure modulate saphenous vein remodeling ex vivo. J Biomech. 2005 Sep;38(9):1760-9.

47 Ramachandra AB, Humphrey JD, Marsden AL. Gradual loading ameliorates maladaptation in computational simulations of vein graft growth and remodelling. J R Soc Interface. 2017 May; 14(130):14

48 Prim DA, Potts JD, Eberth JF. Pulsatile Perfusion Bioreactor for Biomimetic Vascular Impedances. ASME. DOI: 10.1115/1.4040648. 